\title{
ESTUDOS DA TRADUÇÃO E LITERATURA COMPARADA: CONFLITO E COMPLEMENTARIDADE
}

\author{
Walter Carlos Costa* \\ Universidade Federal de Santa Catarina/ \\ Universidade Federal do Ceará /CNPq
}

\begin{abstract}
Resumo: Os Estudos da Tradução mantiveram, em seu surgimento, uma relação complexa com a literatura comparada, marcada por conflito e complementaridade, já que muitos de seus fundadores vinham dos estudos literários, e, alguns, da literatura comparada. Nas últimas quatro décadas, podemos distinguir duas tendências principais, ocorridas de forma desigual nas duas disciplinas. De um lado, os Estudos da Tradução experimentaram um grande crescimento, com ampliação de seua objetos de pesquisa e certo afastamento dos estudos literários. Por outro lado, a Literatura Comparada vem se abrindo progressivamente para o fenômeno tradutório e para a própria disciplina dos Estudos da Tradução. O conflito inicial desapareceu e o diálogo ficou restrito a uma parte dos Estudos da Tradução, que poderíamos chamar de Estudos Literários da Tradução, e a Literatura Comparada. Isso ocorre particularmente no Brasil, onde os estudos literários da tradução ocupam um importante espaço nos programas de pós-graduação, sobretudo nos programas específicos de estudos da tradução.
\end{abstract}

Palavras-chave: Estudos da Tradução. Literatura Comparada. Disciplina

\footnotetext{
* Estudou Filologia Românica na Katholieke Universiteit Leuven (Bélgica). Tem Doutorado sobre as traduções de Borges para o inglês pela University of Birmingham, Reino Unido e Pós-Doutorado pela Universidade Federal de Minas Gerais. É professor do Departamento de Língua e Literatura Estrangeiras da Universidade Federal de Santa Catarina. Foi presidente da Abrapt (Associação Brasileira de Pesquisadores em Tradução, na gestão 2010-2013). Atualmente está em colaboração técnica no Departamento de Letras Estrangeiras da Universidade Federal do Ceará. Fortaleza, Ceará, Brasil. E-mail: walter.costa@gmail.com
} 


\title{
TRANSLATION STUDIES AND COMPARATIVE LITERATURE: CONFLICT AND COMPLEMENTARITY
}

\begin{abstract}
Translation Studies had in its beginnings, a complex relationship with Comparative Literature, marked by conflict and complementarity, as many of its founders came from literary studies, and some of Comparative Literature itself. Over the past four decades, we can distinguish two main trends occurring unevenly in both disciplines. On the one hand, Translation Studies experienced a robust growth, with expansion of its research objects and certain detachment of literary studies. On the other hand, Comparative Literature has been gradually opening to the translation phenomenon and the very discipline of Translation Studies. The initial conflict disappeared and the dialogue was restricted to a portion of Translation Studies, which we might call the Literary Translation Studies and Comparative Literature. This is particularly true in Brazil, where literary translation studies occupy an important space in graduate programs, particularly in the specific programs of translation studies.
\end{abstract}

Keywords: Translation Studies. Comparative Literature. Discipline

A disciplina dos Estudos da Tradução teve um desenvolvimento extraordinário nas últimas quatro décadas. Fundada, em grande parte, por pesquisadores oriundos dos estudos literários, a disciplina teve, no início, uma relação complexa com a Literatura Comparada, marcada muitas vezes pelo conflito mas também por complementaridade. Por sua parte, a Literatura Comparada, uma disciplina bem estabelecida, primeiro ignorou a nova disciplina mas, nos últimos anos, tem mostrado uma crescente abertura para a tradução, em geral, e para os Estudos da Tradução como disciplina. Esse desenvolvimento assimétrico das duas disciplinas se reflete nos programas dos congressos e nos manuais: enquanto nos congressos e manuais dos Estudos da Tradução, a Literatura Comparada costuma ser ignorada e os estudos literários ocupam posição minoritária, nos congressos e manuais de Literatura Comparada, a tradução tem aparecido cada vez mais frequentemente.

Em 2009, José Lambert apresentou, na PGET (Pós-Graduação em Estudos da Tradução), da UFSC, por ocasião de sua primeira permanência como professor visitante durante um semestre, com 
bolsa de Professor Visitante do CNPq, um quadro pessimista em relação à literatura comparada, disciplina em que atuou com destaque durante décadas ${ }^{1}$. Para Lambert, que foi um dos fundadores e é uma das figuras destacadas da disciplina dos Estudos da Tradução, a literatura comparada padece de uma falha estrutural, ligada às suas origens. Como a literatura comparada surgiu, segundo ele, na esteira do fortalecimento dos Estados nacionais e da expansão colonialista no século XIX, ela manteria um viés nacional e monolíngue, o que a impediria de ser realmente internacional e considerar o plurilinguismo inerente à experiência literária de todos os tempos e países. Também devido a essa limitação de tipo nacionalista, a literatura comparada não conseguiria incorporar a tradução entre suas categorias e, em consequência, trata a literatura traduzida como se fosse produzida no idioma do país e as relações interculturais como se a operação tradutória acontecesse sem maiores dificuldades e não oferecesse maior interesse teórico e crítico.

Esse desconforto com os parâmetros da literatura comparada levou-o a se interessar cada vez mais pela literatura traduzida. Ao examinar o papel da tradução nas literaturas nacionais - sobretudo na França, Bélgica e Alemanha - Lambert foi percebendo que a tradução desempenhava um papel muito mais importante do que apenas configurar as diferentes literaturas, às vezes de forma auxiliar, às vezes de forma central. Isso explica seu progressivo interesse, ao longo dos anos, pelo papel da tradução na configuração e no funcionamento da sociedade e de algumas de suas instituições, como a universidade e, ultimamente, as empresas. Com o estabelecimento da disciplina dos Estudos da Tradução, Lambert foi se distanciando da tradução literária stricto sensu e se aproximando, de fato, de outras disciplinas que se desenvolveram na mesma época, como os Estudos Culturais. Atualmente, se interessa particularmente pela interface entre os estudos da tradução e os estudos organizacionais.

Em 2010, a revista Scientia Traductionis, publicou uma extensa entrevista com Lambert, em que ele reconstrói, com riqueza de detalhes, a história da disciplina. ${ }^{2}$ Ele sublinha, em vários momentos, 
o fato que os Estudos da Tradução foram tomando cada vez mais uma feição não-literária, e não por acaso mas intencionalmente, lembrando que a questão tinha sido levantada em um primeiro momento por Yves Gambier e tratada por ele próprio no artigo "Is Translation Studies too Literary?"3.

Os manuais e as programações dos congressos da área refletem essa mudança programática claramente. Assim, a primeira edição do livro Translation Studies, de Susan Basnett, de 1980, fala de tradução em geral mas os exemplos citados são quase todos literários e pertencentes ao universo europeu e norte-americano. Sua segunda edição, de 1991, inclui referências a Israel, China, América Latina e o mundo árabe, assim como às teorias feministas de tradução e à tradução de teatro. Quando surge a terceira edição, em 2002, está claro que o manual de Bassnett já não reflete os últimos desenvolvimentos da área, que tinha tomado outros rumos, rumos fundamentalmente não-literários e que incluem cada vez mais os estudos da interpretação. Sintomaticamente, um dos grandes repertórios bibliográficos da área, do Departamento de Tradução e Interpretação da Universidad de Alicante, e que contém cerca de 38.000 itens, se chama Bibliografía de Interpretación y Traducción (http:// aplicacionesua.cpd.ua.es/tra_int/usu/buscar.asp?idioma=es).

Discourse and Translation, de Basil Hatim e Ian Mason, de $1990^{4}$, é uma obra essencialmente de linguística textual e o mesmo se pode dizer de In other words ${ }^{5}$, de Mona Baker, de 1992, em que a literatura brilha pela ausência. Mona Baker, aliás, se tornaria, nas décadas seguintes, um dos grandes nomes da área, montando sua própria editora, a St. Jerome Press, e editando a influente Encyclopedia of Translation Studies, publicada pela Routledge em 1998 e reeditada em 20096. Nessa enciclopédia, a literatura aparece pouco e só tem maior peso nos verbetes históricos. Um dos poucos verbetes dedicados especificamente à tradução literária é "Tradução literária - questões de pesquisa", de autoria de José Lambert. Os outros são: "tradução literária”, "antologias literárias", "poéticas da tradução", "tradução de poesia" e "tradução de Shakespeare". 
Uma das coletâneas que visa a oferecer um panorama da disciplina Estudos da Tradução por especialistas, é $A$ Companion to Translation Studies, organizado por Piotr Kuhiwczak and Karin Littau, e publicado pela Multilingual Matters em 2007 . O livro tem um capítulo intitulado "Literary Translation", escrito por Theo Hermans, um dos teóricos da chamada "escola da manipulação", em voga nos inícios da disciplina e autor de The Manipulation of Literature: Studies in Literary Translation (1985) ${ }^{8}$. Hermans, que é professor de literatura holandesa e literatura comparada no University College, de Londres, oferece um panorama dos estudos da tradução literária nos estudos da tradução, que ele afirma ter sido dominado por três correntes: "linguística, funcionalismo e intervencionismo". As duas primeiras correntes estão bastante distantes dos estudos literários, enquanto a última, em que ele agrupa autores como Bassnett, Venuti e Berman, além das feministas, se caracterizariam por dar importância aos aspectos ideológicos. Essa corrente, sim, mantém um diálogo com correntes similares nos estudos literários mas não especialmente com a literatura comparada.

Os dois volumes do Handbook of Translation Studies, editado por Yves Gambier e Luc van Doorslaer em 2011 ${ }^{9}$, reservam um lugar bastante modesto para a tradução literária, pulverizada em uns poucos itens como "Children's literature and translation", "Drama translation", "Literary studies and translation studies", "Domestication and foreignization", "Literary Translation", "Poetry Translation", "Stylistics and translation".

O mesmo ocorre com The Oxford Handbook of Translation Studies, editado por Kirsten Malmkjær and Kevin Windle em 2012 $2^{10}$, em que a imensa maioria dos verbetes tem a ver com a tradução não-literária e com a interpretação.

A Companion to Translation Studies, de Sandra Bermann \& Catherine Porter, publicado em $2014^{11}$, representa uma nota dissonante em relação aos manuais anteriores, ao dedicar um grande bloco à relação entre tradução e literatura comparada, mas uma literatura comparada com incorporação da tradução, em uma perspectiva de literatura mundial. O capítulo se intitula, significativa- 
mente, "Translation and Comparative World Literature" e vai da página 347 à página 436. Além disso, a tradução literária aparece em várias outras rubricas das outras partes do livro.

Finalmente, um livro recém-publicado divide, no próprio título, o espaço de pesquisa entre tradução e literatura comparada: Degrees of Affinity Studies in Comparative Literature and Translation, de Zuoliang Wang ${ }^{12}$. Cabe notar que o livro é financiado pelo Chinese Fund for the Humanities and Social Sciences, que tem por objetivo divulgar a produção chinesa de ciências humanas em inglês. Embora a tradução esteja onipresente no livro, o enfoque é de história literária e de literatura comparada e a disciplina "estudos da tradução" não é mencionada sequer uma vez sequer em todo o texto.

Lieven D’Hulst, que pode ser considerado um discípulo de José Lambert, no artigo "Comparative Literature versus Translation Studies: Close Encounters of the Third Kind?", publicado na European Review, em $2007^{13}$, defende uma convergência entre Literatura Comparada e Estudos da Tradução.

Necessitamos de métodos para integrar o estudo da tradução na literatura comparada. Será que isso significaria que a tradução deveria se tornar o núcleo da literatura comparada como alguns, como Emily Apter, têm defendido ultimamente? ${ }^{14}$

D'Hulst cita a seguinte passagem de Translation zone, de Emily Apter, publicado em 2006:

Ao tentar repensar os paradigmas críticos para as humanidades depois do 11 de setembro, com sua ênfase em língua e guerra, o problema da criolização e o mapeamento da "in-tradução" das línguas, deslocamentos no cânone mundial e nos mercados literários, e o impacto das novas tecnologias de tradução da informação, tentei imaginar um programa para uma nova literatura comparada usando a tradução como fulcro. ${ }^{15}$ 
que ele comenta da seguinte forma:

Isto pode vir a acontecer, se suficientes pessoas compartilharem essa crença. A crítica ao monolinguismo e ao estado-nação pode ser apoiada pela tradução mas talvez a questão não seja se devemos ou não "usar" a tradução mas como deveríamos estudar a tradução. Colocando a questão de forma direta: qual é o espaço que um novo programa de literatura comparada está disposto a aceitar para os estudos da tradução como uma disciplina autônoma? Os estudos da tradução se tornaram uma disciplina bem estabelecida na academia e parece ser capaz de manter seu estatuto interdisciplinar por um tempo. Por que deveria a literatura comparada relutar em reconhecer o aparato conceitual e a metodologia que foi desenvolvida para o estudo da tradução? ${ }^{16}$

D’Hulst não diz com todas as letras, mas o que ele está defendendo é a metodologia dos estudos descritivos da tradução, que ele próprio aplicou, como José Lambert, à história da tradução literária na França e que parece não ter tido o impacto esperado nos círculos da literatura comparada e da historiografia literária.

Susan Bassnett é muito mais radical em sua avaliação, embora também pregue uma convergência entre estudos da tradução e literatura comparada. Ela, que, justamente, tinha defendido a ascensão dos estudos da tradução e a redução da literatura comparada a um setor dos estudos da tradução, faz uma autocrítica feroz no artigo "Reflections on Comparative Literature in the Twenty-First Century", publicado em $2006^{17}$ na revista Comparative Critical Studies:

Em 1993 eu publiquei um livro sobre literatura comparada, no qual eu dizia que a disciplina estava à beira da morte. A base de minha argumentação era que os debates sobre uma pretensa crise na literatura comparada provinham de 
uma herança do positivismo do século XIX e o fracasso em reconhecer as implicações políticas dos processos de transferência intercultural. Isso tinha levado, no Ocidente, a uma sensação de decadência da disciplina, embora em ou-tras partes do mundo a literatura comparada estivesse florescendo. Eu disse que talvez tivesse chegado o momento de uma nova disciplina mais autoconfiante, a disciplina emergente dos estudos da tradução, ocupar o palco. "A literatura comparada teve sua importância como disciplina. O trabalho transcultural em estudos femininos, em teoria pós-colonial, em estudos culturais. Mudou a face dos estudos literários. Teríamos que considerar os estudos da tradução a partir de agora como a disciplina principal e a literatura comparada como uma área importante mas subsidiária. ${ }^{18}$

Bassnett acrescenta que essa postura era deliberadamente provocativa e tentava promover a nova disciplina tanto quanto declarar morta a antiga. O balanço que ela faz não é favorável aos estudos da tradução:

Hoje, reexaminando minha proposta, vejo que ela aparece fundamentalmente equivocada: os estudos da tradução não se desenvolveram muito nas últimas três décadas e a comparação permanece no centro da maioria das pesquisas de estudos da tradução. O que eu diria hoje, se voltasse a escrever aquele livro, é que nem a literatura comparada nem os estudos da tradução devem ser vistos como disciplinas: as duas são muito mais métodos de enfocar a literatura, modos de ler que são mutuamente benéficos. A crise na literatura comparada derivava de um prescritivismo excessivo combinado com metodologias culturalmente específicas que não podiam ser aplicadas universalmente ou que não eram universalmente relevantes. ${ }^{19}$

No final de seu artigo, que é bastante ambivalente, Bassnett defende uma espécie de confluência entre as disciplinas, destacando, 
como D’Hulst, a importância da tradução nos estudos de literatura comparada e sublinhando "a necessidade de colocar a história da tradução no centro de todo estudo de literatura comparada" ${ }^{20}$.

Chama a atenção que tanto Basnnett como D'Hulst estejam falando nesses textos para os colegas de literatura comparada, não para os colegas dos estudos da tradução.

De fato, a convergência entre estudos da tradução (ou, pelo menos, da tradução) e literatura comparada tem acontecido mais na área de literatura comparada do que na de estudos da tradução. Isso se reflete nos eventos, em que a programação costuma incluir uma minoria de conferências e mesas-redondas sobre tradução literária. No entanto, no Brasil, os estudos da tradução literária parecem fortes, tanto nos eventos dos Estudos da Tradução como nos eventos de Literatura Comparada. Assim, no XI Congresso Internacional da ABRAPT e V Congresso Internacional de Tradutores, realizado na Universidade Federal de Santa Catarina, em Florianópolis, de 23 a 26 de setembro de 2013, uma porção significativa de simpósios foram dedicados à tradução literária, refletindo a pesquisa que se faz efetivamente no país (ver o Caderno de Resumos do evento: https:// abrapt.files.wordpress.com/2013/11/abrapt-2013-resumos.pdf.).

Por outro lado, nos congressos da ABRALIC (Associação Brasileira de Literatura Comparada), que são os maiores eventos da área dos estudos literários no país, a tradução tem ocupado um espaço cada vez maior. Assim, o próximo XIV Congresso, que ocorrerá em Belém, PA, de 29 de junho a 3 de julho de 2015, a tradução está presente não apenas nos simpósios mas faz parte do próprio lema do congresso: "Fluxos e correntes: trânsitos e traduções literárias".

Finalmente, cabe ressaltar que há uma área em que os Estudos da Tradução e a Literatura Comparada parecem convergir: a historiografia da tradução. Estão em curso, em vários países, projetos de história da tradução. Entre eles, cabe destacar a monumental The Oxford History of Literary Translation in English ${ }^{21}$, e a não menos ambiciosa Histoire des traductions en langue française $e^{22}$. Nesses projetos, que provavelmente mudarão a história literária 
dos países de língua inglesa e francesa, os pesquisadores envolvidos costumam trabalhar com um arsenal teórico e metodológico tanto dos estudos literários como dos estudos da tradução.

\section{Notas}

1. Comunicação "Littérature comparée et étude des traductions: Pour une reconnaissance de la dynamique des littératures", apresentada na mesa-redonda " $\mathrm{Li}$ teratura Comparada, Estudos da Tradução e Teoria Literária”, em 06/08/2009.

2. "Interview with José Lambert", by Gustavo Althoff \& Lilian Fleuri. Scientia Traductionis, n. 7, 2010, pp. 207-234. Disponível em https://periodicos.ufsc.br/ index.php/scientia/article/download/1980-4237.2010n7p207/12890, acesso em 20/04/15.

3. Génesis. Revista cientifica do ISAI. Tradução e Interpretação, 2005, 5: 20

4. Hatim, Basil \& Mason, Ian. Discourse and the Translator. Harlow: Longman, 1990.

5. Baker, Mona. In Other Words: A Coursebook on Translation, London \& New York: Routledge, 1992.

6. Baker, Mona \& Saldanha, Gabriela (eds.) The Routledge Encyclopedia of Translation Studies, $2^{\text {nd }}$, revised and extended edn. London: Routledge, 2009.

7. Kuhiwczak, Piotr and Karin Littau (eds). A Companion to Translation Studies, Clevedon: Multilingual Matters, 2007.

8. Hermans, Theo (ed.) The Manipulation of Literature. Studies in Literary Translation, London \& Sydney: Croom Helm, 1985. 
9. Gambier, Yves \& Van Doorslaer, Luc. Handbook of Translation Studies. Volumes I \& II. Amsterdam/Philadelphia: Benjamins, 2010 \& 2011.

10. Malmkjær, Kirsten \& Windle, Kevin. The Oxford Handbook of Translation Studies. Oxford: Oxford University Press, 2012.

11. Bermann, Sandra \& Porter, Catherine. A Companion to Translation Studies. Oxford: Wiley, 2014.

12. Wang, Zuoliang. Degrees of Affinity Studies in Comparative Literature and Translation. Berlin: Springer, 2015.

13. European Review, Vol. 15, No. 1, 95-104 (2007).

14. "And we need adequate methods to integrate the study of translation into comparative literature. Does all this mean that translation should become the heart of comparative literature, as some like Emily Apter have recently pleaded for?", p. 9.

15. "In attempting to rethink critical paradigms in the humanities after 9/11, with special emphasis on language and war, the problem of creolization and the mapping of languages 'in-translation', shifts in the world canon and literary markets, and the impact of enhanced technologies of information translation, I have tried to imagine a program for a new comparative literature using translation as a fulcrum." E. Apter (2005) The Translation Zone: A New Comparative Literature. Princeton: Princeton University Press, 2005, p. 256.

16. "This may eventually happen, if enough people share such a belief. The criticism of monolingualism and of nation-states may well be supported by translation, but maybe the question is not as much whether we should or not 'use' translation, but how we should study translation. Putting it bluntly: how much space for translation studies as a discipline in its own right is a new programme for comparative literature ready to accept? Translation studies has become a fully established discipline in academia and seem capable of upholding its interdisciplinary status for a while. Why should comparative literature be reluctant when it comes to recognizing the conceptual apparatus and methodology that has been developed for the study of translation?", p. 9. 
17. Comparative Critical Studies 3, 1-2, pp. 3-11, 2006.

18. "In 1993 I published a book on comparative literature in which I argued that the subject was in its death throes. The basis of my case was that debates about a so-called crisis in comparative literature stemmed from a legacy of nineteenth-century positivism and a failure to consider the political implications of intercultural transfer processes. This had led, in the West, to a sense of the subject being in decline, though elsewhere in the world comparative literaure, albeit under other labels, was flourishing. I argued that perhaps the time had come for a more self-confident discipline, the emergent discipline of translation studies to take centre stage: 'Comparative literature as a discipline has had its day. Cross-cultural work in women's studies, in post-colonial theory, in cultural studies has changed the face of literary studies generally. We should look upon translation studies as the principle discipline from now own, with comparative literature as a valued but subsidiary subject area'”, p. 6.

19. "Today, looking back at that proposition, it appears fundamentally flawed: translation studies has not developed very far at all over three decades and comparison remains at the heart of much translation studies scholarship. What I would say were I writing the book today is that neither comparative literature nor translation studies should be seen as a discipline: rather both are methods of approaching literature, ways of reading that are mutually beneficial. The crisis in comparative literature derived from excessive prescriptivism combined with distinctive culturally specific methodologies that could not be universally applicable or relevant, p. 6 .

20. "the need to position the history of translation centrally within any comparative literary study", p. 10.

21. Até agora foram publicados os seguintes volumes: Ellis, Roger. The Oxford History of Literary Translation in English Volume 1: To 1550. Oxford: Oxford University, 1980; Braden, Gordon; Cummings, Robert; \& Gillespie, Stuart. The Oxford History of Literary Translation in English Volume 2 1550-1660. Oxford: Oxford University Press, 2010; Gillespie, Stuart \& Hopkin, David. The Oxford History of Literary Translation in English Volume 3: 1660-1790. Oxford: Oxford University Press, 2005; France, Peter \& Haynes, Kenneth. The Oxford History of Literary Translation in English Volume 4: 1790-1900. Oxford: Oxford University Press, 2010.

22. Apareceram até agora dois volumes, consagrados respectivamente, ao século XIX e aos séculos XVII e XVIII: Chevrel, Yves, D’Hulst, Lieven \& Lombez, 
Christine. Histoire des traductions en langue française. XIXe siècle 1815-1914. Lagrasse: Verdier, 2012 ; e Chevrel, Yves; Cointre, Annie \& Tran-Gervat, Yen-Maï. Histoire des traductions en langue française, XVIIe et XVIIIe siècles (1610-1815). Lagrasse: Verdiers, 2014.

Recebido em: 01/09/2014

Aceito em: 26/ 09/2014 